\title{
The internet-community response to the Mexican Earthquake of February 1, 2019
}

\author{
Alexandr $V$. Tertyshnikov ${ }^{1}$ \\ E.K. Fedorov Institute of Applied Geophysics, Moskow, Russia
}

\begin{abstract}
Signs of the threat of the Mexican earthquake of February 1,2019 , are presented according to estimates of the recurrence of query words in Internet electronic publications. These estimates (information signals) have been determined using the Internet search engines.

Before the earthquake, an increase in the recurrence of query words semantically related to seismic-hazard precursors was revealed in electronic publications relative to the seismically hazardous region. After the earthquake, the activity of the Internet community decreases.
\end{abstract}

\section{Introduction}

The concept of environment includes both natural environment and its anthropogenic surroundings. The society as an element of anthropogenic surroundings strongly affects the environment state and is a biological indicator of its variations. Geophysical-field disturbances in a seismically active region with a seismotectonic anomaly (STA) affect the activity in the behavior of bioindicators - primary transformers of geophysical disturbances (Fig. 1).

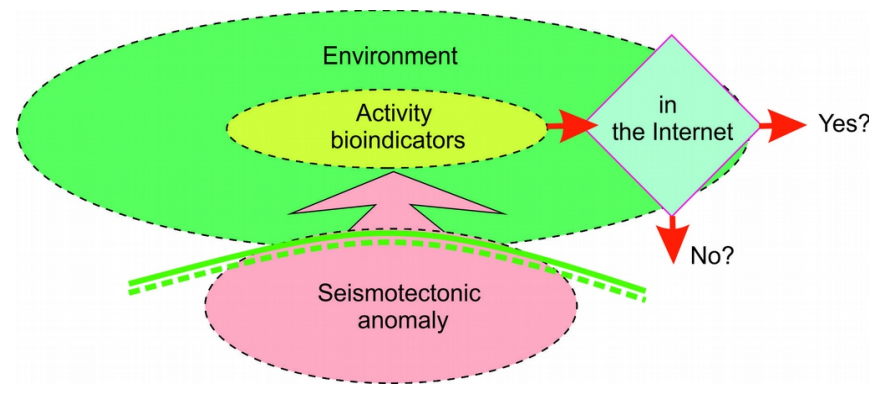

Fig. 1 - Experiment on reginal seismic-hazard diagnostics.

The behavior of bioindicators is directed towards searching for support of their observations and feelings and towards their description and discussion through electronic messages and texts on the Internet. The internet community makes it possible to intensify

\footnotetext{
1* Corresponding author: atert@mail.ru
} 
the occurrence of weak signals/feelings of individual users and to increase their number and activity in repeating discussed words semantically related to earthquake precursors and earthquake-development processes. Changes in the behavior of people are manifested in their increased activity in the form of psychomotor actions, communications, search for information, including that on the Internet.

Measurements of social activity using the Internet search engines are used in different fields of human activity [1-4]. An analysis of the recurrence of chosen words using the Internet search engines will make it possible to estimate the presence of seismogenic geophysical-field disturbances caused by a developing STA.

\section{Problem statement}

Both linguistic-search and data-analysis programs have been developed for word processing in the Internet search engines. These programs use sets of queries associated with the ontology of the processes under study. Taking into account geospheric interactions and experience in monitoring the precursors of hazardous natural phenomena are necessary to diagnose the symptoms of environmental disturbances in seismically hazardous regions with STAs.

It is necessary to have the time series of recurrence of query words for the seismically hazardous region under study in searching for information precursors of earthquakes on the Internet. Experience in working with search engines has confirmed the presence of regional differences in the activity of the internet community before earthquakes.

The simplest simulation model of a box with inlets, outlets, control Federal Standart $\mathrm{R}$ ISO/MEK 122-7-99 actions, and positive feedback may be used to describe internal interactions and regularities in the behavior of the internet community in a seismically hazardous region (rhomb in Fig.1).

Advances in social networks, internet, and artificial-intelligence technologies furnish a unique opportunity to diagnose variations in the social behavior of different groups of people on a time scale close to real for concrete seismically hazardous regions. To solve this problem, it is necessary to regularly collect and analyze available information on characteristics associated with the state of natural environment and potential precursors of violent earthquakes hazardous to human life.

A lot of criteria are used in the internet counters provided by search engines. For the experiment on the estimation of the activity of the internet community in responding to a seismic hazard, in a first approximation, it is sufficient to use the frequency of the occurrence of preselected key query words on the Internet before and after the violent earthquake in Japan. After preliminary studies, the words Air, Health, and Water were selected as query words.

It is sufficient to limit the time span by three weeks before and after the earthquake. It is also necessary to take into account the background activity of the recurrence of query words for the region under analysis not to consider the national features of variations in information flows as earthquake precursors.

The results of keyword recurrences found by search engines were tabulated together with accompanying texts, in which the query word was found by the search engines and the geographical reference of the texts and messages to the region under analysis was taken into account.

The obtained time series on the number of visits to electronic texts on the Internet before and after violent earthquakes may be used to estimate the relation with a seismic hazard. 


\section{Experiment results}

On February 1, 2019, 16:14:12.2 UTC, in southeastern Mexico (14.86 N; $92.20 \mathrm{~W})$, an earthquake $(\mathrm{Mw}=6.6)$ occurred (Chiapas, Mexico). Its seismic center was in the Chiapas State in the vicinity of the Guatemala border, and its hypocenter was at a depth of $67.9 \mathrm{~km}$. Earthquake shocks were pronounced in many regions of Mexico, Guatemala, El Salvador, and Belize.

The earthquakes preceding and accompanying the Mexican earthquake according to archive data of the Geophysical Service of the Russian Academy of Sciences:

1) January 8, 2019, 12:39:29.4, $30.57 \mathrm{~N}, 131.09 \mathrm{E}, \mathrm{H}=41 \mathrm{~km}, \mathrm{M}=6.1$ (according to other estimates 6.3, 6.5, Kyushu, Japan);

2) January 22, 2019, 5:10:0.6, $9.98 \mathrm{~S}, 119.15 \mathrm{E}, \mathrm{H}=10 \mathrm{~km}, \mathrm{M}=6.3$ (according to other estimates 6.4) and 19:1:41.8, 43.13 S, 42.35 E, $\mathrm{H}=10 \mathrm{~km}, \mathrm{M}=6.6$ (according to other estimates 6.8, 6.3, Sumba region, Indonesia);

3) February 2, 2019, 9:27:33.2, 2.72 S, 100.21 E, H=14 km, M=6.2 (according to other estimates 6.3, 6.0, Southern Sumatera, Indonesia); 9:58:10.7, 2.61 S, $100.28 \mathrm{E}, \mathrm{H}=13 \mathrm{~km}, \mathrm{M}=5.4 ; 10: 59: 29.0,2.74 \mathrm{~S}, 100.28 \mathrm{E}, \mathrm{H}=21 \mathrm{~km}, \mathrm{M}=6.1$ (according to other estimates $6.3,5.8) ; 11: 01: 36.7,2.75 \mathrm{~S}, 100.30 \mathrm{E}, \mathrm{H}=14 \mathrm{~km}$, $\mathrm{M}=6.1$ (according to other estimates 5.8); and 12:04:32.5, $36.44 \mathrm{~N}, 70.77 \mathrm{E}$, $\mathrm{H}=211 \mathrm{~km}$ (mid-focal), $\mathrm{M}=5.8$ (according to other estimates 6.2, Hindu Kush region, Afghanistan);

4) February 8, 2019, 11:55:4.4, 9.85 N, $126.39 \mathrm{E}, \mathrm{H}=11 \mathrm{~km}, \mathrm{M}=6.3$ (according to other estimates 6.4, 5.6, Mindanao, Philippines);

5) February 21, 2019, 12:22:39.6, $42.80 \mathrm{~N}, 141.95 \mathrm{E}, \mathrm{H}=41 \mathrm{~km}, \mathrm{M}=5.9$ (according to other estimates 6.2, 5.4, Hokkaido, Japan).

February 2, 2019, was seismically active. Figure 2 shows the variations in the sum of recurrences of each of the min-max normalized query words ( «Air», «Water», «Health») for the region under consideration.

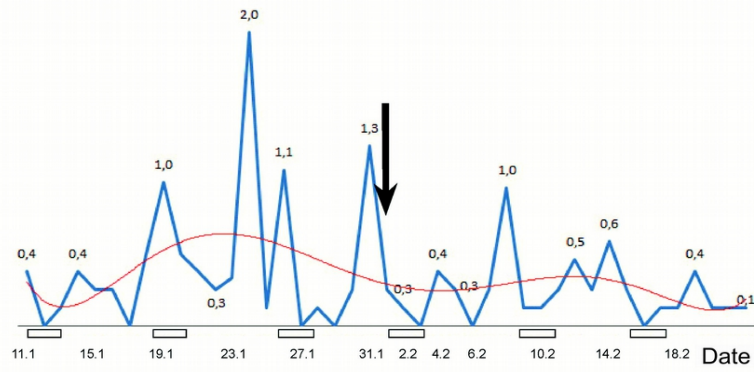

Fig. 2. Variations in the sum of recurrences of each of the min-max normalized query words («Air», «Water», «Health») for the georeference of the Mexico region and with respect to the earthquake of February 1, 2019. The rectangles correspond to Saturday-Sunday and the red curve corresponds to the 6 th-degree polynomial approximation.

After the seismic-calm period before the earthquake, the recurrence of query words increases (two strong signals).

The magnetic disturbance within the time span under analysis was low [5]. The peak event of January 24, 2019, occurred when the magnetic storm was weak with an average daily Kp-index of 3 [6]. However, on February 1-2, 2019, when the value of $\mathrm{Kp}$-index was also 3, there was no peak in the recurrence of query words, because the earthquake had already occurred. The full moon was observed on January 21, 2019. 
The mean over the observation period January 10 - February 22, 2019, amounts to 0.336, the rms deviation is 0.397. The mean over the February 2-22, 2019, observation period after the earthquake amounts to 0.238 , the rms deviation is 0.223 . The mean over the observation period January 10 - February 1, 2019, before the earthquake is almost two times higher: 0.430 and the rms deviation is 0.499 .

The hypothesis of the constancy of variances for the periods before and after the earthquake according to the Fisher ratio test with a significance level of $\alpha<0.05$ is not fulfilled. The time series under analysis is nonstationary. Therefore, in further studies, the methods of parametric statistics should be used with great caution. This is also true for testing the hypothesis of the equality of expected values of the periods under consideration.

When compared to the peak event of January 24, 2019, the amplitude of subsequent peaks keeps within the golden proportion. The weekly periodicity and increased power of both low- and high-frequency variations before the earthquake are manifested in Fig. 2.

The amplitudogram of the time series (Fig. 2) obtained using a sliding window 16 days wide is shown in Fig. 3. This amplitudogram has been constructed using the fast Fourier transform [7-9] and is presented in relative gradations. The calculated spectra are assigned to the right-hand border of the window.

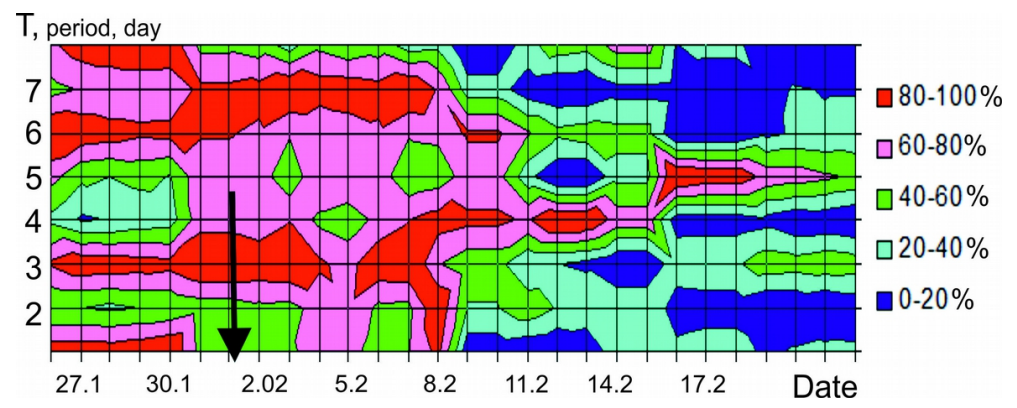

Fig. 3. The amplitudogram of the time series (Fig. 2) according to the sliding window 16 days wide. The black vertical arrow corresponds to the day of the earthquake.

The particular feature of Fig. 3 is that the estimates calculated in spectra for the each-period amplitude were normalized to the minimax of the time span under analysis within the considered observation period.

Morphologically, the amplitudogram includes three fragments-before, after the earthquake, and the stage of relaxation approximately two weeks after the earthquake. This is the response of the internet community to the disastrous event.

Before the earthquake, the amplitudes of 3-day, 6-day, and 8-day periods increase with respect to the mean and the amplitudes of 4-day and 5-day periods decrease with respect to the mean. The multimode distribution of local extrema in the recurrence of the periods under analysis is manifested before the earthquake. The weekly periodicity increases before and after the earthquake.

The earthquake occurred at increased levels of the amplitudes of the rest of periods before February 11, 2019. Then, the amplitudograms were retrieved to background and decreased (when compared to the background) values. The time span after the earthquake is of interest as regards the response of the internet community to the occurred disaster.

An analysis of the activity of regional social networks, including internet publications related to the region under study not only in Russian, but also in 
English, could supplement the results obtained. The major social networks, such as Facebook $^{\mathrm{TM}}$ and Twitter ${ }^{\mathrm{TM}}$ store billions of user reports and simultaneously provide the opportunity to add external videos and images to such reports [3], which may contain seismic-hazard signals.

Using the spectral analysis makes it possible to reveal slight effects within natural noise. Thus, in [10], with the aid of the fast Fourier transform according to the 16-day sliding window, in the periodograms of daily mean data on deceleration of the Monitor-E satellite, it was revealed that the period of seismic calm with minimally powerful short-period deceleration variations with periods of 2 and 3 days was manifested no less than a week before the generalized earthquake hazard with a subsequent increase in the power of these variations. These periods were also pronounced for the considered earthquake in Japan.

\section{Conclusions}

Before the Mexican earthquake of February 1, 2019, an increase in the recurrence of query words semantically associated with precursors of a seismic hazard was revealed in electronic publications related to the seismically hazardous region. After the earthquake, the activity of the internet community decreases.

\section{References}

1. A. Yudin, Search engines in the world, statistics $2018 /$ https://marketer.ua/searchengine-stat-2018/

2. G.G. Gusev et al., Method and server for predicting the popularity of a content element. Patent for an invention. Application: 2015140585., Bull. № 10 (2017).

3. A.A. Lyubushin, Method of dynamic assessment of seismic hazard. Application for invention 2012144388/28 from 18.10.2012, Bull. № 8. (2014)

4. A.V. Tertyshnikov, Earthquake precursors and features of their registration (VIKA, Spb, 1996) (in Russian)

5. A.V. Tertyshnikov, Seismic-ozones effects and the problem of earthquake forecasting. (VIKA, Spb, 2000)

6. A.V. Tertyshnikov, Earth Research from space, 2007, № 4. P. 88-91.

7. A.V. Tertyshnikov, Fundamentals of emergency monitoring (Moscow-Obninsk, 2013)

8. A.V. Tertyshnikov, Organization of forecasting natural emergencies (Moscow, 2013)

9. A.V. Tertyshnikov et al., Magnetic indexes. - (Moscow-Obninsk, 2014)

10. A.V. Tertyshnikov, 2015. http://elibrary.ru/item.asp?id=21273666.

11. A.V. Tertyshnikov, Heliogeophysical research, 21, 12-17 (2018) http://vestnik.geospace.ru/index.php?id=526.

12. A.V. Tertyshnikov et al., Heliogeophysical research, 22, 12-17 (2019) http://vestnik.geospace.ru/index.php?id=532. 